\title{
Pain characteristics in amyotrophic lateral sclerosis patients and its impact on quality of life: a prospective observational study in a northern city of China
}

\author{
Zhaohong Kong ${ }^{1}$, Peng Chen ${ }^{2}$, Jian Jiang ${ }^{1}$, Xiaoyun Wang ${ }^{3}$, Ying Wang ${ }^{2}$, Yanghong Shi ${ }^{2}$, Bin Zhao ${ }^{2}$, \\ Jiang $\mathrm{Zhu}^{2}$
}

${ }^{1}$ Department of Neurology, Renmin Hospital of Wuhan University, Wuhan, China; ${ }^{2}$ Department of Neurology, The First Hospital of Yulin, Yulin, China; ${ }^{3}$ Yidu Cloud (Beijing) Technology Co., Ltd., Beijing, China

Contributions: (I) Conception and design: Z Kong, J Zhu, B Zhao; (II) Administrative support: Z Kong, P Chen, Y Wang; (III) Provision of study materials or patients: Z Kong, J Jiang, B Zhao; (IV) Collection and assembly of data: J Zhu, X Wang, Y Shi; (V) Data analysis and interpretation: Z Kong, J Zhu, Y Wang; (VI) Manuscript writing: All authors; (VII) Final approval of manuscript: All authors.

Correspondence to: Jiang Zhu, MD, PhD. Department of Neurology, The First Hospital of Yulin, No. 93 Yuxi Street, Gaoxin District, Yulin, China. Email: zhenbeitai@163.com.

\begin{abstract}
Background Pain in amyotrophic lateral sclerosis (ALS) has a considerable impact on the quality of life of both patients and their caregivers, and thus the identification and evaluation of pain characteristics in ALS should be addressed. However, due to the scarcity of research data, pain in ALS is still frequently underestimated and insufficiently treated. The aim of this study was to investigate the characteristics of pain in patients with ALS using standardized pain questionnaires.

Methods: Eighty-nine patients diagnosed with ALS were interviewed. Consecutive patients with peripheral neuropathy were used as control subjects and were matched to the ALS subjects by age and sex. Patient data including gender, age, the Amyotrophic Lateral Sclerosis Functional Rating Scale-Revised (ALSFRS-R) score, and the pain severity index (PSI) were collected. The characteristics between ALS and peripheral neuropathy, and between ALS patients with and without pain were compared.

Results: In all, 89 patients with ALS and 89 control subjects with peripheral neuropathy were included. There were no significant differences in sex ratio and age between the two groups. There were significantly more patients with pain symptoms in the ALS group (35/89, 39\%) than in the peripheral neuropathy group (20/89, 22\%). Quality of life was significantly affected in the ALS patients with pain (using ALS patients without pain as control subjects).
\end{abstract}

Conclusions: The results suggested that pain was a significant symptom in patients with ALS and had a considerable impact on quality of life.

Keywords: Amyotrophic lateral sclerosis (ALS); pain; enjoyment of life; relationship with other people; severity of disease

Submitted Mar 31, 2020. Accepted for publication Oct 12, 2020.

doi: 10.21037/apm-20-864

View this article at: http://dx.doi.org/10.21037/apm-20-864

\section{Introduction}

Amyotrophic lateral sclerosis (ALS) is a progressive neurodegenerative disorder characterized by the degeneration of primary motor neurons in the brainstem, cortex, and spinal cord. Annually, ALS affects approximately between two and three people per 100,000. Its primary symptoms include muscle weakness and atrophy, spasticity, speech disturbances, dysphagia, and respiratory insufficiencies (1). Thus, for a long time, ALS was considered to only cause damage to the motor neuron without sensory nervous system involvement. 
As a result, up until the past decade, pain in ALS was largely overlooked by clinicians.

Considering the substantial impact on the quality of life of ALS patients and their caregivers, the identification and evaluation of pain in ALS should not be disregarded. Recent guidelines for ALS treatment reported that pain may be present in ALS patients and should be treated (2). However, due to the scarcity of research data, pain in ALS is still frequently underestimated and insufficiently treated. The incidence, degree, sites of pain, and impact on the quality of life of patients with ALS have not been fully addressed. Although there are reports indicating that the frequency of pain in patients with ALS ranges between 15-85\%, the precise prevalence is not clear (3-5), especially considering that pain frequency is varied among different populations (4-6).

With a large aging population in China, there is a pressing need to investigate pain characteristics in the Chinese ALS patients. To our knowledge, studies in the Chinese population are still lacking. Therefore, we performed a prospective study using the Brief Pain Inventory (BPI) questionnaire to describe pain characteristics and its effect on the daily functions of patients. We firstly investigated whether pain was a significant symptom in patients with ALS using patients with peripheral neuropathy as a control group. We then described pain characteristics and its impact on quality of life in ALS individuals. We present the following article in accordance with the STROBE reporting checklist (available at http://dx.doi.org/10.21037/apm-20-864).

\section{Methods}

\section{Patients}

Patients diagnosed with ALS according to the revised El Escorial criteria in The First Hospital of Yulin between January 2010 and June 2018 were potentially suitable for the study (7). The inclusion criteria were as follows: (I) patients with ALS who had a follow-up appointment, and (II) patients who could understand and answer the questionnaire alone or with the assistance of a researcher. The exclusion criteria were as follows: (I) patients with radiculopathy, plexopathy, entrapment neuropathy, or neurological diseases $(8,9)$; (II) patients with atypical motor neuron syndromes (for example, Kennedy's disease) and conditions affecting peripheral nerves; (III) pregnant women (10); (IV) patients unable to give informed consent or understand the questionnaires. Consecutive patients with peripheral neuritis who visited our hospital between January 2010 and June 2018 were used as the control group, and were matched to the ALS subjects by age and sex.

The study was conducted in accordance with the Declaration of Helsinki (as revised in 2013). And this study was approved by the ethics committee of The First Hospital of Yulin (2009-12-01). We obtained written informed consent from each patient or substitute decision-maker.

\section{Procedures}

Patients who agreed to participate in this study were interviewed in person between January 2010 and June 2018. The sample size was calculated using $\mathrm{G}^{*}$ Power software according to a previous study (3-5). The effect size for a primary outcome was the pain frequency in patients with ALS and peripheral neuritis. The patients were asked to answer the BPI and the Amyotrophic Lateral Sclerosis Functional Rating Scale-Revised (ALSFRS-R) questionnaires at a follow-up appointment. The questionnaire was administered by the first author. ALSFRS-R was used to assess the functional score of the patients with ALS. ALSFRS-R contains 12 items, with each scored from 0 (worst) to 4 (best). The total ALSFRS-R score ranges from 0 to 48 , with higher scores indicating greater physical status and function (11).

Pain was evaluated using the Chinese version of BPI, which has been widely used to evaluate pain (12). BPI is a self-administered questionnaire assessing the presence and severity of current pain (presence of pain in the last 24 hours) and its interference with daily activities. The pain scale ranges from 0 (no pain) to 10 (pain as bad as one can imagine). The pain severity index (PSI) was derived by averaging the items of worst pain, average pain, and pain perceived at the interview (12). Pain degree was defined as no pain ( $\mathrm{PSI}=0)$, mild pain ( $\mathrm{PSI} \leq 3)$, moderate pain $(4<$ PSI $\leq 6)$, and severe pain $(6<$ PSI $\leq 10)$ (3). BPI also provides information regarding the pain site. It evaluates the influence of pain on an individual's general activity, sleep, mood, daily work, relationships with other people, walking ability, and enjoyment of life. The corresponding interference scale ranges from 0 (no interference) to 10 (complete interference). Data including gender, age, pain degree, pain site, etc., were collected from all patients.

\section{Statistical analysis}

Data analyses were performed using SPSS 19.0 (IBM Corp., 
Table 1 Clinical characteristics in patients with ALS and patients with peripheral neuropathy

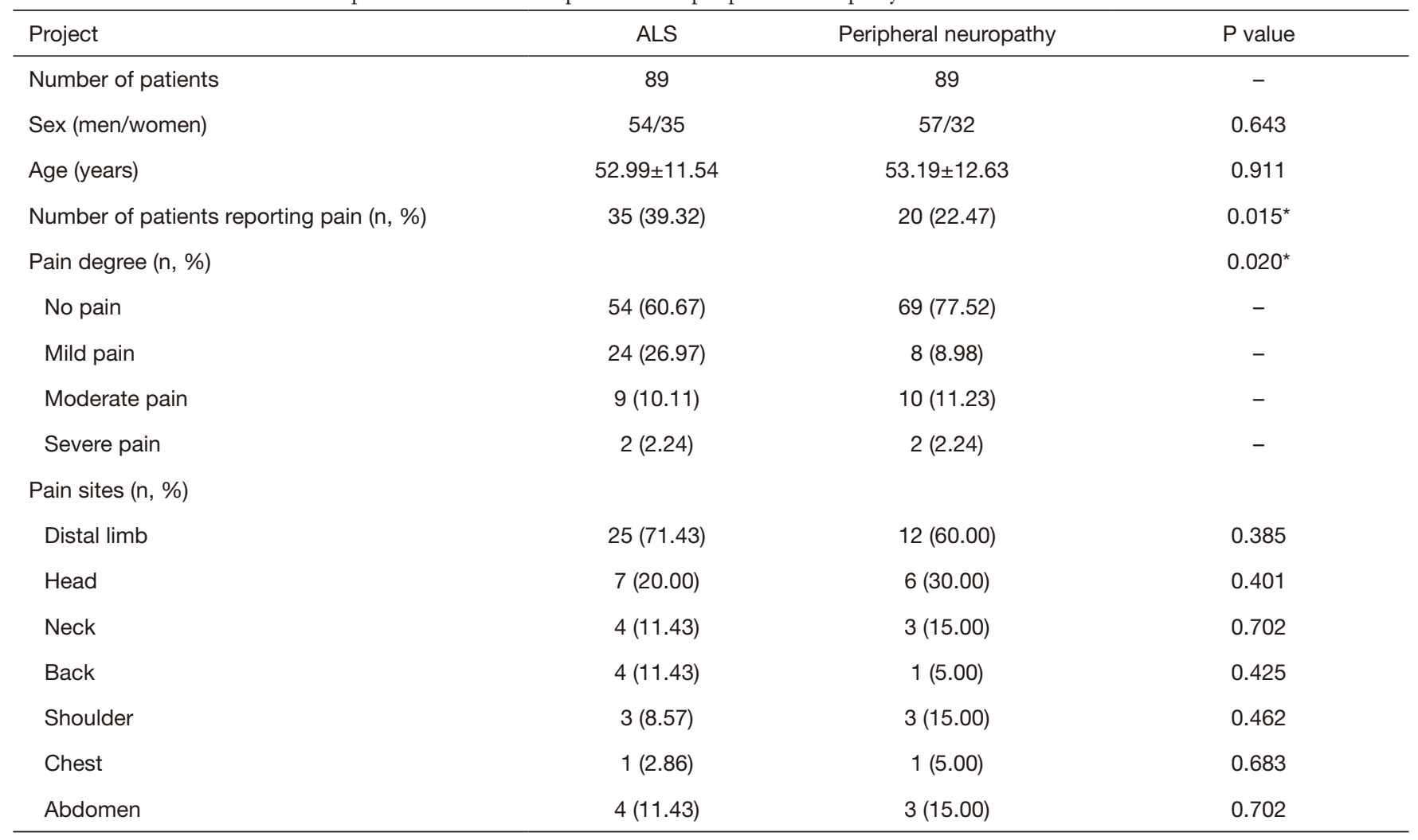

* indicates $\mathrm{P}<0.05$. ALS, amyotrophic lateral sclerosis.

Armonk, NY, USA). Continuous data are presented as mean \pm standard deviation or median/range, and categorical variables are presented as counts or percentages. The variables were compared between the groups (ALS group $v s$. peripheral neuropathy control group; ALS subjects with pain $v s$. ALS subjects without pain; ALS patients with mild pain $v s$. ALS patients with moderate and severe pain) using the independent $t$-test. A P value $<0.05$ indicated statistical significance.

\section{Results}

\section{Baseline data}

All included patients received general pain treatment, including non-steroidal anti-inflammatory drugs (NSAIDs) administered by a doctor. There were no statistically significant differences in the treatment of pain between the two groups. In all, 89 patients with ALS met the inclusion criteria and were enrolled into the study, including 54 males and 35 females (average age: $52.99 \pm 11.54$ years; Table 1 ).
The disease duration from diagnosis in this group ranged from 1 to 144 months. The peripheral neuropathy group included 57 males and 32 females, with an average age of $53.19 \pm 12.63$ years. The disease duration in this group was from 1 to 144 months. There were no significant differences in sex ratio and age between the two groups $(\mathrm{P}>0.05)$.

\section{Characteristics of pain in the ALS group and the peripheral neuropathy group}

Pain symptoms were observed in significantly more patients in the ALS group $(35 / 89,39 \%)$ than in the peripheral neuropathy group $(20 / 89,22 \%)(\mathrm{P}<0.05$, Table 1), suggesting that pain is a significant symptom in patients with ALS. Specifically, there were 24 patients with mild pain, 9 patients with moderate pain, and 2 patients with severe pain in the ALS group; while in the peripheral neuropathy group, there were 8 patients with mild pain, 10 patients with moderate pain and 2 patients with severe pain. Pain was reported in the head, neck, back, shoulder, chest, and abdomen in both groups of patients. Pain was present 
Table 2 Demographic information and interference of pain on daily functions in patients with ALS

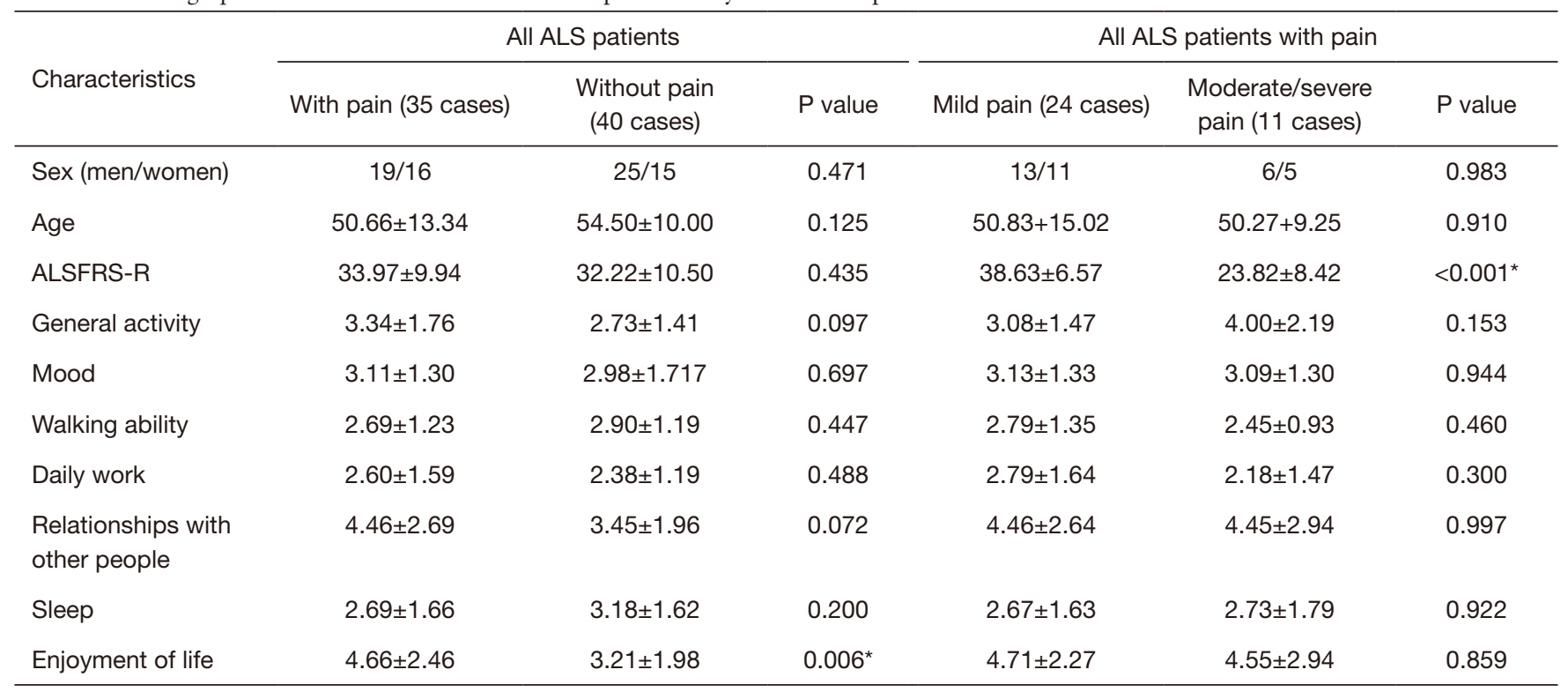

* indicates P<0.05. ALS, amyotrophic lateral sclerosis; ALSFRS-R, Amyotrophic Lateral Sclerosis Functional Rating Scale-Revised.

at more than one site in 8 ALS subjects and 6 peripheral neuropathy subjects. Pain mostly presented in the distal limbs, and there was no significant difference between the two groups $(\mathrm{P}>0.05$; Table 1$)$.

\section{Comparison between ALS patients with pain and ALS patients without pain}

To investigate the impact of pain on quality of life, we compared the interference score derived from the BPI between ALS patients with pain and ALS patients without pain. There were no statistically significant differences in sex, age, and ALSFRS-R between these two groups of patients. The interference score on enjoyment of life was significantly higher in ALS patients with pain compared to ALS patients without pain. As for the interference score on general activity, mood, walking ability, daily work, relationships with other people, and sleep, there were no significant differences between the two groups $(\mathrm{P}>0.05)$.

We also compared the BPI interference scores between ALS patients with mild pain (24 cases) and those with moderate and severe pain (11 cases) to determine whether higher pain degree has a worse impact on quality of life. Results showed that there was no statistical difference in the interference scores on general activity, mood, walking ability, daily work, relationship with other people, sleep, or enjoyment of life between the two groups (Table 2).

\section{Discussion}

Pain in patients with ALS is often overlooked, and research in the Chinese population is lacking. In this study, we found that pain is a significant symptom that may potentially have a deleterious impact on the quality of life of patients with ALS. In our study, the frequency of pain in the ALS group was $39 \%$, which was higher than that of the control group. This is in accordance with the reported pain incidence of $15-85 \%$ (13). We used the BPI questionnaire, which is most frequently used in studies that have evaluated pain in ALS $(3,6,14)$. However, the present study population is a clinicbased sample, and thus the results of our study may not be as generalizable as a population-based study.

As for pain sites, patients with ALS reported pain in various sites, including the back, shoulders, chest, abdomen, and head. In some patients, pain was present at more than one site, with the distal limbs being the most frequently reported site. Some previous studies on ALS also reported that pain could involve the back, as well as both distal and proximal sites in the upper and lower extremities, among others $(3,6,13,14)$. However, Truini et al. (15) and Dalla et al. (16) investigated small nerve fiber loss and reported pain symptoms predominantly in the feet, which suggested that pain sites in ALS might be biased according to different study populations.

The mechanism for pain in ALS is not fully understood. We speculate that it is probably due to the growth of the 
axon buds, which might lead to increased motor nerve unit excitability (3). In ALS patients suffering from skin pain (in the limbs), poor physical activity and muscle atrophy cannot guarantee effective reflux of blood, thus resulting in venous vasodilatation and swelling pain (17). Musculoskeletal pain may occur in later stages because of stress on bones and joints following atrophy and subsequent loss of muscular sheath. Also, numerous studies reported that pain is less common in the bulbar form of ALS, suggesting that skeletal muscle involvement might be a risk factor $(6,18,19)$. Furthermore, lower back pain can also be triggered by uncomfortable positioning. Some other causes of pain include arthralgias, cramps and spasticity due to degenerative joint disease, and skin pressure caused by immobility.

The general enjoyment of life in the ALS patients was significantly decreased, which was in accordance with the findings of Isak et al. (8). We did not observe any notable difference in general activity, mood, walking ability, daily work, relationships with other people, and sleep between ALS patients with pain and ALS patients without pain, which differed from the study conducted by Wallace et al. (5). They reported that pain significantly affects the quality of life of ALS subjects including activity levels, mood, sleep, and relationships with other people. We believe that this might be attributable to the different study designs, the degree of pain in the target patients, and different controls.

Also, in the present study, we found no statistical difference in the interference scores on daily functions between ALS patients with mild pain and those with moderate and severe pain. The studies of Chiò et al. (6), Rivera et al. (19), Ganzini et al. (20), and Raheja et al. (21) reported that pain appeared more frequently in the later stages of the disease and was related to functional impairment, while Wallace et al. reported that pain frequency was significantly different between the early and late phases (5). These differences are mainly due to the dissimilar study designs and confounding factors included in those studies.

There are some limitations in this study that should be noted. Firstly, the sample size was relatively small for a subgroup analysis. Secondly, this is a prospective study design, and therefore, the course of pain over a longer timeframe was unavailable. Thirdly, this study is lacked a description of pain types, a formal evaluation of depression, and medications for pain treatment. In future, we will conduct a multicenter study to investigate the association between pain intensity in patients with ALS and the severity of ALS.

\section{Conclusions}

The results of our study indicate that pain is a significant symptom in patients with ALS and has a considerable impact on quality of life. We suggest that clinicians pay careful attention to the pain symptoms, standardize the assessment of pain, and administer timely therapy to relieve pain, especially in patients with serious ALS.

\section{Acknowledgments}

Funding: This work was supported by The Scientific research bidding project of Shaanxi Administration of traditional Chinese Medicine (LCMS022) and Wu Jieping Medical Foundation to Dr. Zhaohong Kong (number: 320.6750.19092-23).

\section{Footnote}

Reporting Checklist: The authors have completed the STROBE reporting checklist. Available at http://dx.doi. org/10.21037/apm-20-864

Data Sharing Statement: Available at http://dx.doi. org/10.21037/apm-20-864

Peer Review File: Available at http://dx.doi.org/10.21037/ apm-20-864

Conflicts of Interest: All authors have completed the ICMJE uniform disclosure form (available at http:// dx.doi.org/10.21037/apm-20-864). Dr. ZK reports grants from Shaanxi Administration of Traditional Chinese Medicine, grants from Wu Jieping Medical Foundation, outside the submitted work. Dr. PC reports grants from Shaanxi Administration of Traditional Chinese Medicine, grants from Wu Jieping Medical Foundation, outside the submitted work. Dr. JJ reports grants from Shaanxi Administration of Traditional Chinese Medicine, grants from Wu Jieping Medical Foundation, outside the submitted work. Dr. Xiaoyun $W$ and Ying $W$ report grants from Shaanxi Administration of Traditional Chinese Medicine, grants from Wu Jieping Medical Foundation, outside the submitted work. Dr. YS reports grants from Shaanxi Administration of Traditional Chinese Medicine, grants from Wu Jieping Medical Foundation, outside the submitted work. Dr. BZ reports grants from Shaanxi Administration of Traditional Chinese 
Medicine, grants from $\mathrm{Wu}$ Jieping Medical Foundation, outside the submitted work. Dr. JZ reports grants from Shaanxi Administration of Traditional Chinese Medicine, grants from $\mathrm{Wu}$ Jieping Medical Foundation, outside the submitted work.

Ethical Statement: The authors are accountable for all aspects of the work in ensuring that questions related to the accuracy or integrity of any part of the work are appropriately investigated and resolved. The study was conducted in accordance with the Declaration of Helsinki (as revised in 2013). And this study was approved by the ethics committee of The First Hospital of Yulin (2009-12-01). We obtained written informed consent from each patient or substitute decision-maker.

Open Access Statement: This is an Open Access article distributed in accordance with the Creative Commons Attribution-NonCommercial-NoDerivs 4.0 International License (CC BY-NC-ND 4.0), which permits the noncommercial replication and distribution of the article with the strict proviso that no changes or edits are made and the original work is properly cited (including links to both the formal publication through the relevant DOI and the license). See: https://creativecommons.org/licenses/by-nc-nd/4.0/.

\section{References}

1. Mitchell JD, Borasio GD. Amyotrophic lateral sclerosis. Lancet 2007;369:2031-41.

2. Miller RG, Jackson CE, Kasarskis EJ, et al. Practice parameter update: the care of the patient with amyotrophic lateral sclerosis: multidisciplinary care, symptom management, and cognitive/behavioral impairment (an evidence-based review): report of the Quality Standards Subcommittee of the American Academy of Neurology. Neurology 2009;73:1227-33.

3. Hanisch F, Skudlarek A, Berndt J, et al. Characteristics of pain in amyotrophic lateral sclerosis. Brain Behav 2015;5:e00296.

4. Pizzimenti A, Aragona M, Onesti E, et al. Depression, pain and quality of life in patients with amyotrophic lateral sclerosis: a cross-sectional study. Funct Neurol 2013;28:115-9.

5. Wallace VC, Ellis CM, Burman R, et al. The evaluation of pain in amyotrophic lateral sclerosis: a case controlled observational study. Amyotroph Lateral Scler Frontotemporal Degener 2014;15:520-7.
6. Chiò A, Canosa A, Gallo S, et al. Pain in amyotrophic lateral sclerosis: a population-based controlled study. Eur J Neurol 2012;19:551-5.

7. Brooks BR, Miller RG, Swash M, et al. El Escorial revisited: revised criteria for the diagnosis of amyotrophic lateral sclerosis. Amyotroph Lateral Scler Other Motor Neuron Disord 2000;1:293-9.

8. Isak B, Pugdahl K, Karlsson P, et al. Quantitative sensory testing and structural assessment of sensory nerve fibres in amyotrophic lateral sclerosis. J Neurol Sci 2017;373:329-34.

9. Traynor BJ, Codd MB, Corr B, et al. Amyotrophic lateral sclerosis mimic syndromes: a population-based study. Arch Neurol 2000;57:109-13.

10. Verma A, Bradley WG. Atypical motor neuron disease and related motor syndromes. Semin Neurol 2001;21:177-87.

11. Franchignoni F, Mora G, Giordano A, et al. Evidence of multidimensionality in the ALSFRS-R Scale: a critical appraisal on its measurement properties using Rasch analysis. J Neurol Neurosurg Psychiatry 2013;84:1340-5.

12. Hoffman DL, Sadosky A, Dukes EM, et al. How do changes in pain severity levels correspond to changes in health status and function in patients with painful diabetic peripheral neuropathy? Pain 2010;149:194-201.

13. Chiò A, Mora G, Lauria G. Pain in amyotrophic lateral sclerosis. Lancet Neurol 2017;16:144-57.

14. Stephens HE, Lehman E, Raheja D, et al. Pain in amyotrophic lateral sclerosis: Patient and physician perspectives and practices. Amyotroph Lateral Scler Frontotemporal Degener 2015;17:21-9.

15. Truini A, Biasiotta A, Onesti E, et al. Small-fibre neuropathy related to bulbar and spinal-onset in patients with ALS. J Neurol 2015;262:1014-8.

16. Dalla Bella E, Lombardi R, Porretta-Serapiglia C, et al. Amyotrophic lateral sclerosis causes small fiber pathology. Eur J Neurol 2016;23:416-20.

17. Borasio GD, Voltz R. Palliative care in amyotrophic lateral sclerosis. J Neurol 1997;244 Suppl 4:S11-7.

18. Caress JB, Ciarlone SL, Sullivan EA, et al. Natural history of muscle cramps in amyotrophic lateral sclerosis. Muscle Nerve 2016;53:513-7.

19. Rivera I, Ajroud-Driss S, Casey P, et al. Prevalence and characteristics of pain in early and late stages of ALS. Amyotroph Lateral Scler Frontotemporal Degener 2013;14:369-72.

20. Ganzini L, Johnston WS, Hoffman WF. Correlates of suffering in amyotrophic lateral sclerosis. Neurology 1999;52:1434-40. 
21. Raheja D, Stephens HE, Lehman E, et al. Patientreported problematic symptoms in an ALS treatment trial. Amyotroph Lateral Scler Frontotemporal Degener
2016;17:198-205.

(English Language Editors: A. Kassem and J. Gray)

Cite this article as: Kong Z, Chen P, Jiang J, Wang X, Wang Y, Shi Y, Zhao B, Zhu J. Pain characteristics in amyotrophic lateral sclerosis patients and its impact on quality of life: a prospective observational study in a northern city of China. Ann Palliat Med 2021;10(2):1668-1674. doi: 10.21037/apm-20-864 